HU-EP-02/13

\title{
More chiral operators on the lattice
}

\author{
Werner Kerler \\ Institut für Physik, Humboldt-Universität, D-10115 Berlin, Germany
}

\begin{abstract}
Instead of the Ginsparg-Wilson (GW) relation we only require generalized chiral symmetry and show that this results in a larger class of Dirac operators describing massless fermions, which in addition to GW fermions and to the ones proposed by Fujikawa includes many more general ones. The index turns out to depend solely on a basic unitary operator. We use spectral representations to analyze the new class and to obtain detailed properties. We also show that our weaker conditions still lead properly to Weyl fermions and to chiral gauge theories.
\end{abstract}

\section{Introduction}

In a large part of the works on chiral fermions the Dirac operator $D$ is required to satisfy the Ginsparg-Wilson (GW) relation [1]

$$
\left\{\gamma_{5}, D\right\}=\rho^{-1} D \gamma_{5} D
$$

with a real constant $\rho$, and to be $\gamma_{5}$-hermitian,

$$
D^{\dagger}=\gamma_{5} D \gamma_{5} .
$$

Conditions (1.1) and (1.2) imply that the operator $\mathbb{1}-\rho^{-1} D$ is unitary and $\gamma_{5}$-hermitian. Therefore, instead of imposing (1.1) and (1.2) as is done in such works, it is completely equivalent to require $D$ to have the form

$$
D=\rho(\mathbb{1}-V) \quad \text { with } \quad V^{\dagger}=V^{-1}=\gamma_{5} V \gamma_{5} .
$$


In this notation the condition for the generalized chiral symmetry of the classical action of Lüscher [2] in the form used in Ref. [3] reads

$$
\gamma_{5} D+D \gamma_{5} V=0 \quad \text { with } \quad V^{\dagger}=V^{-1}=\gamma_{5} V \gamma_{5}
$$

In the present paper we observe that (1.4) provides a weaker condition than the GW relation (1.1). Accordingly, instead of (1.1) and (1.2) as are usually imposed, we here only require (1.4) and (1.2) and investigate the resulting larger class of Dirac operators.

We note that using (1.2) one gets from (1.4)

$$
D+D^{\dagger} V=0, \quad D^{\dagger}+D V^{\dagger}=0,
$$

by which it follows that $V$ and $D$ commute and that $D$ is normal,

$$
[V, D]=0, \quad D D^{\dagger}=D^{\dagger} D
$$

To account for this we require $D$ to be a function of $V$,

$$
D=F(V)
$$

i.e. to depend on $V$ and possibly on constants, however, not on any other operator.

We show that in addition to GW fermions with (1.1) and to the ones proposed by Fujikawa 44 and their extension [5] the new class includes many more general ones. We nevertheless find that our weaker conditions still lead properly to Weyl fermions and to chiral gauge theories. Thus it becomes obvious that these conditions constitute the more general principle.

In addition to the general conditions (1.4) and (1.2), we require that $F$ allows for a nonvanishing index of the Dirac operator. Our respective analysis is based on spectral representations. The index turns out to depend solely on the basic unitary operator $V$. We introduce spectral functions which on the one hand side allow for a construction, which gives insight into possible forms of $F$, and on the other side describe the location of the spectrum. We also give a realization of the unitary operator $V$ related to our general construction of the Dirac operator $D$.

In Section 2 we obtain fundamental properties of the functions $D=F(V)$. In Section 3 we discuss the special cases of GW fermions, of the ones proposed by Fujikawa (bringing $D$ of them also into the form $F(V)$ ) and of the extension of the Fujikawa type. In Section 4 we develop realizations of subclasses. We first study the one where $F(V)$ can be represented by a power series. We then give a more general construction for operators of the class, which is based on spectral functions. In a next step we further extend this construction applying appropriate functions. We then address the question which sets of $V$ are related to the subclasses and give a realization of $V$ holding for the general construction of $D$. Further, by working out a particular example, the considerable freedom in this construction is illustrated. In Section 5 we show that our conditions still lead to Weyl operators and to chiral gauge theories. Section 6 contains our conclusions. 


\section{Properties from spectral representation}

On the finite lattice $V$ has the spectral representation $V=\sum_{k} v_{k} P_{k}$ with eigenvalues satisfying $\left|v_{k}\right|=1$ and orthogonal projections $P_{k}=P_{k}^{\dagger}$. Therefore the functions $F(V)$ can be represented by $F(V)=\sum_{k} f\left(v_{k}\right) P_{k}$.

Using $\gamma_{5}$-hermiticity we get the more detailed form

$$
V=P_{1}^{(+)}+P_{1}^{(-)}-P_{2}^{(+)}-P_{2}^{(-)}+\sum_{k\left(0<\varphi_{k}<\pi\right)}\left(\mathrm{e}^{i \varphi_{k}} P_{k}^{(\mathrm{I})}+\mathrm{e}^{-i \varphi_{k}} P_{k}^{(\mathrm{II})}\right)
$$

in which the projections satisfy

$$
\gamma_{5} P_{j}^{( \pm)}=P_{j}^{( \pm)} \gamma_{5}= \pm P_{j}^{( \pm)}, \quad \gamma_{5} P_{k}^{(\mathrm{I})}=P_{k}^{(\mathrm{II})} \gamma_{5}
$$

The spectral representation of $D=F(V)$ then becomes

$$
\begin{aligned}
D & =f(1)\left(P_{1}^{(+)}+P_{1}^{(-)}\right)+f(-1)\left(P_{2}^{(+)}+P_{2}^{(-)}\right) \\
& +\sum_{k\left(0<\varphi_{k}<\pi\right)}\left(f\left(\mathrm{e}^{i \varphi_{k}}\right) P_{k}^{(\mathrm{I})}+f\left(\mathrm{e}^{-i \varphi_{k}}\right) P_{k}^{(\mathrm{II})}\right)
\end{aligned}
$$

in which $D$ is characterized by the function $f\left(\mathrm{e}^{i \varphi}\right)$. Clearly this function enters only at the values $\mathrm{e}^{i \varphi}=\mathrm{e}^{i \varphi_{k}}$. However, since we want to define $D$ in general (in particular, for any gauge field configuration), we have to specify $f(v)$ for all $v=\mathrm{e}^{i \varphi}$.

Inserting the general form (2.3) into (1.2) and (1.5) we obtain the conditions

$$
\begin{gathered}
f(v)^{*}=f\left(v^{*}\right), \\
f(v)+f(v)^{*} v=0,
\end{gathered}
$$

respectively, on the functions $f(v)$. These conditions imply

$$
f(1)=0, \quad f(-1) \text { real } .
$$

The operator form of (2.4) is

$$
F(V)^{\dagger}=F\left(V^{\dagger}\right)
$$

In addition to giving $D$ by (2.3), the functions $f(v)$ obviously describe the location of its spectrum. For continuous $f\left(\mathrm{e}^{i \varphi}\right)$ the eigenvalues of $D$ reside on a closed curve in the complex plane which is symmetric to the real axis and meets this axis at zero and at the value $f(-1)$ (which is required to be nonzero below).

Denoting the dimensions of the right-handed and of the left-handed eigenspace for eigenvalue \pm 1 of $V$ by $N_{+}( \pm)$and $N_{-}( \pm)$, respectively, we have from (2.2)

$$
\operatorname{Tr}\left(\gamma_{5} P_{1}^{( \pm)}\right)= \pm N_{ \pm}(1), \quad \operatorname{Tr}\left(\gamma_{5} P_{2}^{( \pm)}\right)= \pm N_{ \pm}(-1), \quad \operatorname{Tr}\left(\gamma_{5} P_{k}^{(\mathrm{I})}\right)=\operatorname{Tr}\left(\gamma_{5} P_{k}^{(\mathrm{II})}\right)=0
$$


With this because of $f(1)=0$, using the resolvent $(D-\zeta \mathbb{1})^{-1}$, we obtain the index of $D$,

$$
\lim _{\zeta \rightarrow 0} \operatorname{Tr}\left(\gamma_{5} \frac{-\zeta}{D-\zeta \mathbb{1}}\right)=\left\{\begin{array}{ll}
N_{+}(1)-N_{-}(1) & \text { for } f(-1) \neq 0 \\
N_{+}(1)-N_{-}(1)+N_{+}(-1)-N_{-}(-1) & \text { for } f(-1)=0
\end{array},\right.
$$

and also find

$$
\lim _{\zeta \rightarrow 0} \operatorname{Tr}\left(\gamma_{5} \frac{D}{D-\zeta \mathbb{1}}\right)=\left\{\begin{array}{ll}
N_{+}(-1)-N_{-}(-1) & \text { for } f(-1) \neq 0 \\
0 & \text { for } f(-1)=0
\end{array} .\right.
$$

Adding up (2.9) and (2.10) the sum on the l.h.s. gets $\operatorname{Tr}\left(\gamma_{5} \mathbb{1}\right)=0$ so that in any case

$$
N_{+}(1)-N_{-}(1)+N_{+}(-1)-N_{-}(-1)=0 .
$$

Because of (2.9) and (2.11), to admit a nonvanishing index we have to impose the condition

$$
f(-1) \neq 0 \text {. }
$$

After having (2.12), according to (2.11) to allow for a nonvanishing index one has also to require that in addition to 1 the eigenvalue -1 of $V$ occurs. The sum rule (2.11) corresponds to the one found in Ref. [6] for the special case of Dirac operators which satisfy the GW relation (1.1).

Using (2.1) with (2.8) we find

$$
\operatorname{Tr}\left(\gamma_{5} V\right)=N_{+}(1)-N_{-}(1)-N_{+}(-1)+N_{-}(-1),
$$

so that with (2.11) we generally get for the index of the Dirac operators $D$

$$
N_{+}(1)-N_{-}(1)=\frac{1}{2} \operatorname{Tr}\left(\gamma_{5} V\right) .
$$

Thus it turns out that solely the operator $V$ enters for the whole class. This generalizes the results obtained in the GW case [7, 2] and in the overlap formalism [8] before.

On the infinite lattice the unitary space, in which the operators act, gets of infinite dimension and, including limit elements, a Hilbert space. Then to the spectral representations (2.1) and (2.3) the continuous parts $\int_{-\pi}^{\pi} \mathrm{e}^{i \varphi} \mathrm{d} E_{\varphi}$ and $\int_{-\pi}^{\pi} f\left(\mathrm{e}^{i \varphi}\right) \mathrm{d} E_{\varphi}$, respectively, are to be added in which the projector function $E_{\varphi}$ is purely continuous. However, the relations for the index do not change since the continuous spectrum does not contribute to them and because the trace Tr for the expressions of interest can still be defined [9].

We note that condition (2.11) reflects a fundamental difference to the case of the AtiyahSinger (AS) Dirac operator [10], for the nonvanishing eigenvalues $\lambda_{\mathrm{AS} j}$ of which one always has

$$
\hat{N}_{+}\left(\lambda_{\mathrm{AS} j}\right)-\hat{N}_{-}\left(\lambda_{\mathrm{AS} j}\right)=0 \quad \text { for } \quad \lambda_{\mathrm{AS} j} \neq 0,
$$

where $\hat{N}_{+}$and $\hat{N}_{-}$are the dimensions of its right-handed and its left-handed eigenspaces, respectively. For zero eigenvalue of the AS Dirac operator one gets

$$
\hat{N}_{+}(0)-\hat{N}_{-}(0)=\operatorname{dim} \operatorname{ker} D_{\mathrm{AS}}^{(+)}-\operatorname{dim} \operatorname{ker} D_{\mathrm{AS}}^{(+) \dagger}
$$


where $D_{\mathrm{AS}}^{(+)}$is the related Weyl operator (the AS Dirac operator being a composition of $D_{\mathrm{AS}}^{(+)}$and $\left.D_{\mathrm{AS}}^{(+) \dagger}=D_{\mathrm{AS}}^{(-)}\right)$. Thus to obtain a nonvanishing index there it is inevitable to allow the space structure itself to vary and to get chirally asymmetric, which implies its dependence on the particular gauge field configuration. In contrast to this on the lattice there is no such dependence, which is made possible by having (2.11).

\section{GW-type special cases}

\subsection{Ginsparg-Wilson relation}

The Dirac operators satisfying the GW relation (1.1) and (1.2) are the simplest special case of the class defined by (1.4) and (1.2). For these operators $F$ has the form (1.3). As in the more general cases, given the function $F(V)$, suitable $V$ remain to be determined. Thus looking for solutions of the GW relation (1.1), from the present point of view means to look for the appropriate set of operators $V$.

The overlap Dirac operator of Neuberger [11] is well-known to be of form (1.3) and to provide correct results. The underlying principle of the construction of $V$ there is by the normalization $-X\left(\sqrt{X^{\dagger} X}\right)^{-1}$ of an operator $X$, where in that case $X$ is the Wilson-Dirac operator. Another type of construction occurs in an example of Chiu [12], in which $V$ can be identified as the Cayley transform $V=-(Y-i \mathbb{1})(Y+i \mathbb{1})^{-1}$ of a suitable hermitian operator $Y$. This, however, because bounded $Y$ do not allow to reach the eigenvalue -1 of $V$, can provide proper topological properties only in the limit [9]. Thus for the study of theoretical issues, where an explicit construction is desirable, up to now one has to rely on the normalization method.

We note that operators $D$ satisfying the general GW relation [1]

$$
\left\{\gamma_{5}, D\right\}=2 D \gamma_{5} R D \quad \text { with } \quad[R, D] \neq 0
$$

(where $R^{\dagger}=R$ and $\left[\gamma_{\mu}, R\right]=0$ ) do not belong to the class. In fact, with $\gamma_{5}$-hermiticity of $D$ and $\left[\gamma_{5}, R\right]=0$ from this relation one gets $\left[D, D^{\dagger}\right]=2 D^{\dagger}[R, D] D^{\dagger}$. Thus for $[R, D] \neq 0$ the Dirac operator $D$ is not normal, which is in contrast to what is required in (1.6).

In this context it is to be mentioned that for the general GW relation (3.1) the analysis of the index gets rather subtle. One obtains the relation

$$
\operatorname{Tr}\left(\gamma_{5}\left(P_{j}+R Q_{j}\right)\right)+\operatorname{Tr}\left(\gamma_{5} R D\right)=0
$$

in which the projections $P_{j}$ need not to be orthogonal and where eigennilpotents $Q_{j}$ can occur if the dimensions of the respective algebraic and geometric eigenspaces differ. In a lenghty proof [13] it has been shown that for the eigenvalue $\lambda_{k}=0$ of $D$ these dimensions

are equal so that $Q_{k}=0$ and $P_{k}=P_{k}^{\dagger}$. Thus for zero eigenvalue the unwanted term with $Q_{k}$ disappears and $P_{k}$ gets orthogonal as needed. 


\subsection{Proposal of Fujikawa}

The Dirac operators proposed by Fujikawa [4] satisfy

$$
\left\{\gamma_{5}, \gamma_{5} D\right\}=2 a_{0}^{2 k+1}\left(\gamma_{5} D\right)^{2 k+2}, \quad k=0,1,2, \ldots .
$$

They are normal [14], $D^{\dagger} D=D D^{\dagger}$. One may also consider them as satisfying the general GW relation with $R=a_{0}^{2 k+1}\left(D D^{\dagger}\right)^{k}$, where, however, in contrast to (3.1) one has $[R, D]=0$. Using $\gamma_{5}$-hermiticity one gets from (3.3)

$$
D+D^{\dagger}=2 a_{0}^{2 k+1}\left(D^{\dagger} D\right)^{k+1} .
$$

Comparing this with (1.5), $D+D^{\dagger} V=0$, it follows that

$$
V=1-2 a_{0}^{2 k+1} D\left(D^{\dagger} D\right)^{k}=1-2 a_{0}^{2 k+1} D\left(\gamma_{5} D\right)^{2 k},
$$

which obviously is $\gamma_{5}$-hermitian and using (3.4) can be checked to be indeed unitary. With this it follows that (1.4) is fulfilled so that the operators proposed in Ref. [4] are a special case of our general class.

For $D$ one obtains from (3.5)

$$
D=a_{0}^{-1}\left(\frac{1}{2}\right)^{1 /(2 k+1)} \gamma_{5}\left(\gamma_{5}(1-V)\right)^{1 /(2 k+1)},
$$

which is the form of Ref. 沺. The operator $V$ here is given by the normalization

$$
V=-D_{\mathrm{w}}^{(2 k+1)}\left(\sqrt{D_{\mathrm{w}}^{(2 k+1) \dagger} D_{\mathrm{w}}^{(2 k+1)}}\right)^{-1}
$$

of the generalized Wilson-Dirac operator

$$
D_{\mathrm{W}}^{(2 k+1)}=\left(\frac{1}{2} \sum_{\mu} \gamma_{\mu}\left(\nabla_{\mu}-\nabla_{\mu}^{\dagger}\right)\right)^{2 k+1}+\left(\frac{r}{2} \sum_{\mu} \nabla_{\mu}^{\dagger} \nabla_{\mu}\right)^{2 k+1}+m^{2 k+1},
$$

where $\left(\nabla_{\mu}\right)_{n^{\prime} n}=\delta_{n^{\prime} n}^{4}-U_{\mu n} \delta_{n^{\prime}, n+\hat{\mu}}^{4}$ and $-2 r<m \leq 0$. For $k=0$ obviously (3.6) becomes the overlap Dirac operator of Neuberger [11].

The form (3.6), involving in addition operators $\gamma_{5}$, is not yet the one which according to our considerations should occur. To get the latter we note that because of $D^{\dagger}=-D V^{\dagger}$ we can also write (3.5) as

$$
V=1-2 a_{0}^{2 k+1}(-V)^{-k} D^{2 k+1}
$$

With this we obtain

$$
D=a_{0}^{-1}\left(\frac{1}{2}(1-V)(-V)^{k}\right)^{1 /(2 k+1)},
$$

where of the $(2 k+1)$-th roots the one which satisfies (2.7) is to be choosen. Thus with (3.10) we have indeed the form $D=F(V)$ required by the general concept, which appears more convenient in practice, too. 
The functions $f$ of the spectral representation (2.3) for $D$, in the present case are obtained from (3.10) as

$$
f\left(\mathrm{e}^{i \varphi}\right)=a_{0}^{-1} \mathrm{e}^{i(\varphi-\pi) / 2}\left(\sin \frac{\varphi}{2}\right)^{1 /(2 k+1)}
$$

where of the $(2 k+1)$-th roots the real one is to be taken. They give the curves describing the location of the eigenvalues of $D$, which for $k>0$ arise as deformations of the circle for $k=0$. All of them meet the real axis at zero and at the value $f(-1)=a_{0}^{-1}$.

\subsection{Extension of Fujikawa type}

Recently an extension of the proposal of Fujikawa has been reported [5], the Dirac operators of which satisfy

$$
\left\{\gamma_{5}, \gamma_{5} D\right\}=2 a_{1} D^{\dagger} D \Phi\left(a_{1}^{2} D^{\dagger} D\right)
$$

where the operator function $\Phi$ is subject to $\Phi(X)^{\dagger}=\Phi(X)$ for $X^{\dagger}=X$. From the identity $\left[\gamma_{5}, D^{\dagger} D\right]=\left[\left\{\gamma_{5}, \gamma_{5} D\right\}, \gamma_{5} D\right]$ with (3.12) since $D^{\dagger} D=\left(\gamma_{5} D\right)^{2}$ one gets

$$
\left[\gamma_{5}, D^{\dagger} D\right]=0 \text {. }
$$

With this one has $D^{\dagger} D=\gamma_{5} D^{\dagger} D \gamma_{5}$ and finds

$$
\left[D^{\dagger}, D\right]=0 \text {. }
$$

Using $\gamma_{5}$-hermiticity of $D$ to write $(\overline{3.12})$ in the form $D+D^{\dagger}=2 a_{1} D^{\dagger} D \Phi\left(a_{1}^{2} D^{\dagger} D\right)$ and comparing with the basic relation (1.5), $D+D^{\dagger} V=0$, it follows that

$$
V=1-2 a_{1} D \Phi\left(a_{1}^{2} D^{\dagger} D\right) .
$$

Because of (3.13) and $\Phi\left(a_{1}^{2} D^{\dagger} D\right)^{\dagger}=\Phi\left(a_{1}^{2} D^{\dagger} D\right)$ this is seen to be $\gamma_{5}$-hermitian and using (3.14) and (3.12) it can be checked to be unitary. It thus turns out that the operators satisfying (3.12) are also a special case of our general class.

With (3.15) and (1.5) one gets the equation

$$
2 a_{1} D \Phi\left(-a_{1}^{2} V^{-1} D^{2}\right)+V=1
$$

for $D$ and $V$, the solution of which gives $D=F(V)$.

\section{Realizations of subclasses}

\subsection{Expansion in powers of $V$}

We first realize functions $D=F(V)$ of the class in terms of expansions $D=\sum_{\nu} c_{-\nu} V^{\nu}$. According to (2.7) the coefficients $c_{\nu}$ must be real and (1.5) requires them to satisfy $c_{\nu-1}=-c_{-\nu}$. We thus obtain

$$
D=\sum_{\nu \geq 0} c_{\nu}\left(V^{-\nu}-V^{\nu+1}\right)
$$


for which $f(1)=0$ is seen to hold. Condition (2.12) now gets the form

$$
f(-1)=2 \sum_{\nu \geq 0}(-1)^{\nu} c_{\nu} \neq 0
$$

Obviously (1.3) is a special case of (4.1) and arises by putting $c_{\nu}=\rho \delta_{\nu 0}$. Other choices of the coefficients are seen not to allow GW-type relations.

For the functions $f$ in the spectral representation of the Dirac operators (4.1) we obtain the form

$$
f\left(\mathrm{e}^{i \varphi}\right)=2 \mathrm{e}^{i(\varphi-\pi) / 2} \sum_{\nu \geq 0} c_{\nu} \sin (2 \nu+1) \frac{\varphi}{2} .
$$

The functions $\mathrm{e}^{i(\varphi-\pi) / 2} \sin (2 \nu+1) \varphi / 2$ in (4.3) describes rosettes in the complex plane. The eigenvalues of $D$ thus reside on a closed curve which is given by a linear combination of rosette functions and which meets the real axis at zero and at the value (4.2).

In case of an infinite number of terms of the expansion (4.1), convergence properties can be studied considering the Fourier series $\sum_{\nu \geq 0} c_{\nu} \sin (2 \nu+1) \frac{\varphi}{2}$ in (4.3). Uniform convergence then is guaranteed by the condition

$$
\sum_{\nu \geq 0}\left|c_{\nu}\right|<\infty
$$

Having $F(V)$ it remains to specify $V$. Below we show that the overlap $V$ is appropriate here, for which the explicit form $V=-D_{\mathrm{W}}^{(1)}\left({\sqrt{D_{\mathrm{W}}^{(1) \dagger} D_{\mathrm{W}}^{(1)}}}^{-1}\right.$ with $D_{\mathrm{W}}^{(1)}$ given by (3.8) is available. In principle this could also be done for other $V$ suitable in the GW case (1.3), since with respect to the tests considered they should be equivalent to the overlap $V$.

The continuum limit of the propagator gives us further conditions on the coefficients $c_{\nu}$. To study the propagator we note that in the free case and infinite volume with the Fourier representation $V_{\kappa^{\prime} \kappa}=\tilde{V}(\kappa) \delta_{\kappa^{\prime} \kappa}^{4}$ on gets for the powers of $V$ with $\kappa_{\mu}=a p_{\mu}$

$$
\tilde{V}^{-\nu}(\kappa)-\tilde{V}^{\nu+1}(\kappa) \rightarrow a \frac{2 \nu+1}{|m|} i \sum_{\mu} \gamma_{\mu} p_{\mu} \quad \text { for } \quad a \rightarrow 0
$$

while at the corners of the Brillouin zone one has

$$
\tilde{V}^{-\nu}-\tilde{V}^{\nu+1}=2(-1)^{\nu}
$$

According to (4.1) and (4.5) for the usual normalization of the continuum propagator

$$
\sum_{\nu \geq 0}(2 \nu+1) c_{\nu} \rightarrow|m| a^{-1} \quad \text { for } \quad a \rightarrow 0
$$

is needed, while with (4.1) and (4.6) suppression of doublers requires

$$
2 \sum_{\nu \geq 0}(-1)^{\nu} c_{\nu} \rightarrow \infty \quad \text { for } \quad a \rightarrow 0
$$


It is seen that (4.2) is a prerequisite for (4.8). In the special case $c_{\nu}=\rho \delta_{\nu 0}(4.7)$ is satisfied putting $\rho=|m| a^{-1}$, by which (4.8) gets $2|m| a^{-1}$ and is fulfilled, too. In the general case a simple way to satisfy (4.7) and (4.8) as well as (4.2) and (4.4) is to put

$$
c_{\nu}=|m| a^{-1} \hat{c}_{\nu}
$$

and to require

$$
\sum_{\nu \geq 0}(2 \nu+1) \hat{c}_{\nu}=1, \quad \sum_{\nu \geq 0}(-1)^{\nu} \hat{c}_{\nu} \neq 0, \quad \sum_{\nu \geq 0}\left|\hat{c}_{\nu}\right|<\infty
$$

Obviously also weaker conditions than this can be formulated.

With respect to topological charge and chiral anomaly nothing remains to be shown. The reason for this is that only $V$ enters the relation for the index (2.14) and that the overlap $V$ is safely known to give the correct limit of the topological charge (see Ref. [15] for a proof and a discussion of literature). Thus the correct results are also guaranteed here.

\subsection{Construction with spectral functions}

We next give a more general construction of Dirac operators of the class using the functions $f$ of the spectral representation (2.3) as a tool. We start noting that condition (2.5), $f\left(\mathrm{e}^{i \varphi}\right)+f\left(\mathrm{e}^{i \varphi}\right)^{*} \mathrm{e}^{i \varphi}=0$, can be written as

$$
\left(i \mathrm{e}^{-i \varphi / 2} f\left(\mathrm{e}^{i \varphi}\right)\right)^{*}=i \mathrm{e}^{-i \varphi / 2} f\left(\mathrm{e}^{i \varphi}\right) .
$$

This shows that $f$ is of form

$$
f\left(\mathrm{e}^{i \varphi}\right)=\mathrm{e}^{i(\varphi-\pi) / 2} g(\varphi), \quad g(\varphi) \text { real } .
$$

Then from condition (2.4), $f\left(\mathrm{e}^{i \varphi}\right)^{*}=f\left(\mathrm{e}^{-i \varphi}\right)$, the requirement

$$
g(\varphi)=-g(-\varphi)
$$

follows. Further, with $(2 \pi)$-periodicity in $\varphi$ of $f\left(\mathrm{e}^{i \varphi}\right)$, the function $g(\varphi)$ has to satisfy

$$
g(\varphi+2 \pi)=-g(\varphi) .
$$

We now see that forms of $D=F(V)$ can be obtained by determining functions $g(\varphi)$ which are real, odd, and satisfy (4.14).

With the indicated requirements the basic building blocks for the construction of $g$ are the functions $\sin \frac{\nu \varphi}{2}$ and $\cos \frac{\mu \varphi}{2}$ with integer $\nu$ and $\mu$. The conditions on $g$ are fulfilled by $\sin \frac{\nu \varphi}{2}$ with odd $\nu$ and $\sin \frac{\nu \varphi}{2} \cos \frac{\mu \varphi}{2}$ with even $\nu$ and odd $\mu$, where the latter form, however, can be expressed by terms of former type. Further, after multiplication with a function 
of the $\cos \frac{\mu \varphi}{2}$ with even integers $\mu$ one has still the required properties. Thus, with the notation

$$
s_{\nu}=\sin (2 \nu+1) \frac{\varphi}{2}, \quad t_{\mu}=\cos \mu \varphi, \quad \nu, \mu \text { integer }
$$

we arrive at the form

$$
g=\sum_{\nu} s_{\nu} w_{\nu}\left(t_{1}, t_{2}, \ldots\right)
$$

where the $w_{\nu}$ are real functions. Because of the identity

$$
s_{\nu}=s_{0}\left(1+2 \sum_{\mu=1}^{\nu} t_{\mu}\right)
$$

(4.16) can be simplified to

$$
g=s_{0} w\left(t_{1}, t_{2}, \ldots\right)
$$

with a real function $w$. Further, since the $t_{\nu}$ are given by polynomials of $t_{1}$,

$$
t_{2 \mu}=d_{2 \mu} t_{1}^{2 \mu}+d_{2 \mu-2} t_{1}^{2 \mu-2}+\ldots+d_{0}, \quad t_{2 \mu+1}=d_{2 \mu+1} t_{1}^{2 \mu+1}+d_{2 \mu-1} t_{1}^{2 \mu-1}+\ldots+d_{1} t_{1} ，
$$

(4.16) can be cast into the still simpler form

$$
g=s_{0} w\left(t_{1}\right) .
$$

Condition (2.12), $f(-1)=g(\pi) \neq 0$, in case of (4.20) is seen to require

$$
w(-1) \neq 0 .
$$

Further, $w\left(t_{1}\right)$ should, of course, be finite in the region of interest.

The form (4.20) essentially extends the one found in Subsection 4.1 to the case where no series expansion in $V$ is available. To see this one has to note that there according to (4.3) we have

$$
g=2 \sum_{\nu \geq 0} c_{\nu} s_{\nu}=s_{0}\left(b_{0} / 2+\sum_{\mu \geq 1} b_{\nu} t_{\nu}\right)=s_{0} w\left(t_{1}\right)
$$

where the second form follows inserting (4.17) into the first one and introducing coefficients $b_{\mu}=2 \sum_{\nu \geq \mu} c_{\nu}$. Then using (4.19) from the second one the last one is obtained, which is (4.20) specialized to the case where $w\left(t_{1}\right)$ is given by a power series.

The operator $D$ for the construction (4.12) with (4.20),

$$
f\left(\mathrm{e}^{i \varphi}\right)=\mathrm{e}^{i(\varphi-\pi) / 2} s_{0} w\left(t_{1}\right)=\frac{1}{2}\left(1-\mathrm{e}^{i \varphi}\right) w(\cos \varphi),
$$

inserting (4.23) into (2.3) becomes

$$
D=\frac{1}{2}(\mathbb{1}-V) W\left(\frac{1}{2}\left(V+V^{\dagger}\right)\right),
$$

where the operator function $W$ has to satisfy

$$
W(X)^{\dagger}=W\left(X^{\dagger}\right), \quad W(-P) \neq 0 \text { for } P=P^{2}=P^{\dagger}>0 .
$$


To check the continuum limit it remains to consider the operator $W\left(\frac{1}{2}\left(V+V^{\dagger}\right)\right)$ which as compared to the GW case (1.3) replaces the constant $2 \rho$. This can again be done with the overlap $V$. For $\frac{1}{2}\left(V+V^{\dagger}\right)$ in the free case and infinite volume we get with $\kappa_{\mu}=a p_{\mu}$

$$
\frac{1}{2}\left(\tilde{V}(\kappa)+\tilde{V}^{\dagger}(\kappa)\right) \rightarrow 1 \quad \text { for } \quad a \rightarrow 0,
$$

while at the corners of the Brillouin zone we have

$$
\frac{1}{2}\left(\tilde{V}+\tilde{V}^{\dagger}\right)=-1
$$

Thus the requirements (4.7) and (4.8) generalize to

$$
\tilde{W}(1) \rightarrow 2|m| a^{-1}, \quad \tilde{W}(-1) \rightarrow \infty \quad \text { for } \quad a \rightarrow 0 .
$$

These conditions can be satisfied by putting

$$
\tilde{W}=2|m| a^{-1} \hat{W}, \quad \hat{W}(1)=1, \quad \hat{W}(-1) \neq 0,
$$

though again also weaker conditions can be formulated.

With respect to the meaning of these conditions we note that the eigenvalues of $V$ in the free case are those of $\tilde{V}$,

$$
\mathrm{e}^{i \varphi}=-\left(\tau \pm i \sqrt{s^{2}}\right) / \sqrt{\tau^{2}+s^{2}}
$$

where

$$
s^{2}=\sum_{\mu} \sin \kappa_{\mu}^{2}, \quad \tau=m+r \sum_{\mu}\left(1-\cos \kappa_{\mu}\right), \quad-2 r<m<0 .
$$

The real eigenvalues in (4.30) obviously occur for $\kappa_{\mu}=0, \pi$ and one gets +1 if all $\kappa_{\mu}=0$ and -1 at each corner of the Brillouin zone. Noting that

$$
\cos \varphi=\frac{1}{2}\left(\tilde{V}(\kappa)+\tilde{V}^{\dagger}(\kappa)\right)=-\tau / \sqrt{\tau^{2}+s^{2}},
$$

the conditions on $\tilde{W}(1)$ and $\tilde{W}(-1)$ are seen to be related to the behavior of $V$ at its eigenvalues +1 and -1 , repectively.

\subsection{Extension of spectral construction}

To extend the construction of the preceeding Section we note that given a function $g$ with the required properties, then $h(g)$ is again a function with such properties provided that $h$ is odd and real,

$$
h(-x)=-h(x), \quad h(x)^{*}=h(x) \text { for real } x .
$$

With this the form (4.20) generalizes to

$$
g=h\left(s_{0} w\left(t_{1}\right)\right) .
$$


Now condition (4.21) extends to

$$
h(w(-1)) \neq 0
$$

which is satisfied anticipating the requirement of strict monotony of $h$ implied by (4.52).

Looking for functions $h$ an important observation is that because of the identity

$$
s_{0}^{2 k+1}=s_{0} \sum_{\nu=0}^{k}\left(\begin{array}{c}
2 k+1 \\
\nu
\end{array}\right)(-1)^{\nu}\left(1+2 \sum_{\mu=1}^{k-\nu} t_{\nu}\right), \quad k=0,1,2, \ldots
$$

(4.34) reduces to the form (4.20) if $h(x)$ is an integer power, is a polynomial, or allows an expansion in powers of $x$. This greatly restricts nontrivial choices of $h(x)$, i.e. ones by which (4.34) gives something beyond (4.20).

An example of nontrivial choices in this sense, which can be expressed in an elementary way, is

$$
h(x)=x^{1 /(2 k+1)}, \quad k=1,2, \ldots
$$

where the real one of the $(2 k+1)$-th roots is to be taken. One should note that with this one has a different nontrivial choice for each $k$.

The nontrivial choices of $h$ actually are equivalence classes, i.e. equivalent ones are not to be counted as different. For example, to (4.37) the form $h(x)=x^{(2 k+1)} r(x)$ with $r(-x)=r(x)$ is equivalent, because with $r(x)=r(|x|)$ and $\left|s_{0}\right|=\sqrt{\frac{1}{2}\left(1-t_{1}\right)}$ it gives the same form in (4.34) as (4.37). Also forming in addition odd powers because of (4.36) gives nothing new.

The number of nontrivial choices of $h(x)$ which can be explicitly expressed in terms of elementary functions appears to be limited. We give a further explicit example in Subsection 4.7 and also point out strategies for more general constructions there.

The operator $D$ for the construction (4.12) with (4.34),

$$
f\left(\mathrm{e}^{i \varphi}\right)=\mathrm{e}^{i(\varphi-\pi) / 2} h\left(s_{0} w\left(t_{0}\right)\right)
$$

inserting (4.38) into (2.3) becomes

$$
D=-i V^{\frac{1}{2}} H\left(\frac{1}{2 i}\left(V^{\frac{1}{2}}-V^{-\frac{1}{2}}\right) W\left(\frac{1}{2}\left(V+V^{\dagger}\right)\right)\right),
$$

where $V^{\frac{1}{2}}$ is defined with $+\mathrm{e}^{i \varphi_{k} / 2}$ in its spectral representation, $W$ has to satisfy 4.25 and for the function $H$ one needs

$$
H(-X)=-H(X), \quad H(X)^{\dagger}=H(X) \text { for } X^{\dagger}=X
$$

Eq. (4.39) demonstrates the drastic increase of possible forms of Dirac operators which occurs as compared to the GW case. 


\subsection{Example $h(x)=x^{1 /(2 k+1)}$}

To check the continuum limit for a particular choice of a nontrivial $h$, we use (4.37) as an example, where $h$ is conveniently represented in terms elementary functions. With it (4.12) becomes

$$
f\left(\mathrm{e}^{i \varphi}\right)=\mathrm{e}^{i(\varphi-\pi) / 2}\left(s_{0} w\left(t_{1}\right)\right)^{1 /(2 k+1)}=\frac{1}{2}\left(1-\mathrm{e}^{i \varphi}\right)^{1 /(2 k+1)} w(\cos \varphi)^{1 /(2 k+1)} .
$$

The operator $D$ inserting (4.41) into (2.3) and putting $W^{1 /(2 k+1)}=W_{k}$ becomes

$$
D=\left(\frac{1}{2}(1-V)(-V)^{k}\right)^{1 /(2 k+1)} W_{k}\left(\frac{1}{2}\left(V+V^{\dagger}\right)\right)
$$

where of the $(2 k+1)$-th roots the one which satisfies (2.7) is to be choosen and where $W$ is subject to (4.25).

Obviously (4.42) generalizes our form (3.10) of Fujikawa's proposal replacing the constant $a_{0}^{-1}$ there by a function $W_{k}\left(\frac{1}{2}\left(V+V^{\dagger}\right)\right)$. Using $V$ given by (3.7) with (3.8), in the free case and infinite volume one gets with $\kappa_{\mu}=a p_{\mu}$

$$
\tilde{V}(\kappa) \rightarrow 1-\left(\frac{a}{|m|}\right)^{2 k+1}\left(i \sum_{\mu} \gamma_{\mu} p_{\mu}\right)^{2 k+1} \quad \text { for } \quad a \rightarrow 0,
$$

while at the corners of the Brillouin zone one has $\tilde{V}=-1$. The requirements (4.28) thus generalize to

$$
\tilde{W}_{k}(1) \rightarrow 2^{1 /(2 k+1)}(-1)^{k}|m| a^{-1}, \quad \tilde{W}_{k}(-1) \rightarrow \infty \quad \text { for } \quad a \rightarrow 0 .
$$

This can be satisfied by putting

$$
\tilde{W}_{k}=2^{1 /(2 k+1)}(-1)^{k}|m| a^{-1} \hat{W}_{k}, \quad \hat{W}_{k}(1)=1, \quad \hat{W}_{k}(-1) \neq 0,
$$

or by some weaker condition. In the special case where $W_{k}$ is replaced by the constant $a_{0}^{-1}$ according to (4.45) one gets巴

$$
a_{0}^{-1}=2^{1 /(2 k+1)}(-1)^{k}|m| a^{-1} .
$$

The eigenvalues of $V$ in the free case here are

$$
\mathrm{e}^{i \varphi}=-\left(\tau_{k} \pm i \sqrt{\left(s^{2}\right)^{2 k+1}}\right) / \sqrt{\tau_{k}^{2}+\left(s^{2}\right)^{2 k+1}},
$$

with

$$
s^{2}=\sum_{\mu} \sin \kappa_{\mu}^{2}, \quad \tau_{k}=m^{2 k+1}+\left(r \sum_{\mu}\left(1-\cos \kappa_{\mu}\right)\right)^{2 k+1}, \quad-2 r<m<0 .
$$

\footnotetext{
1 Starting from (3.6) as in literature the factor $(-1)^{k}$ also arises by properly calculating $\gamma_{5}\left(\sum_{\mu} \gamma_{\mu} s_{\mu}\right)^{-(2 k+1)}=(-1)^{k}\left(\gamma_{5} \sum_{\mu} \gamma_{\mu} s_{\mu}\right)^{-(2 k+1)}$ before taking the $(2 k+1)$-th root.
} 
In (4.47) the eigenvalues also get +1 if all $\kappa_{\mu}=0$ and -1 at each corner of the Brillouin zone. Noting that

$$
\cos \varphi=\frac{1}{2}\left(\tilde{V}(\kappa)+\tilde{V}^{\dagger}(\kappa)\right)=-\tau_{k} / \sqrt{\tau_{k}^{2}+\left(s^{2}\right)^{2 k+1}}
$$

the conditions on $\tilde{W}(1)$ and $\tilde{W}(-1)$ are again seen to be related to the behavior of $V$ at its eigenvalues +1 and -1 , repectively.

\subsection{Comparison of conditions on $H$ and $W$}

The example of the Dirac operator (4.42) in Subsection 4.4 contains the operator related to the GW relation (1.1), Fujikawa's operator (3.6), and our constructions (4.1) and (4.24) (where (4.1) has been seen to be the expansion version of (4.24)) as special cases. Thus it allows us to compare the features of the continuum limit for $V$ from (3.7) with (3.8) (the case with more general $V$ being considered in Subsection 4.6).

The behavior of $V$ is essentially determined by the choice of $h(x)$, which for $h(x)=$ $x^{1 /(2 k+1)}$ is given by $(4.43)$,

$$
\tilde{V}(\kappa) \rightarrow 1-\left(\frac{a}{|m|}\right)^{2 k+1}\left(i \sum_{\mu} \gamma_{\mu} p_{\mu}\right)^{2 k+1} \quad \text { for } \quad a \rightarrow 0 .
$$

This makes the form for $k>0$ as well as for $k=0$ explicit and illustrates the impact of the function $H$ (which is more generally seen in (4.56) ).

With respect to the condition on $W$, which leads to the usual normalization of the continuum propagator, one has from (4.44),

$$
\tilde{W}_{k}(1) \rightarrow 2^{1 /(2 k+1)}(-1)^{k}|m| a^{-1} \quad \text { for } \quad a \rightarrow 0,
$$

which as well as the suppression of doublers is guaranteed by (4.45). In Fujikawa's case $W_{k}=a_{0}^{-1}$ is a constant, (4.51) becomes $a_{0}^{-1}=2^{1 /(2 k+1)}(-1)^{k}|m| a^{-1}$ and (4.45) is trivially satisfied. In case of the GW relation (1.1), where $W=a_{0}^{-1}=2 \rho$, this reduces to $\rho=$ $|m| a^{-1}$. Thus the straightforward connection to the special cases becomes obvious and it is seen that the essential requirements are the same ones in all cases.

\subsection{Related sets of $V$}

Clearly the determination of the functions $F(V)$ and the selection of the operators $V$ are distinct issues. Nevertheless they are not completely independent. For the choice $h(x)=x$ the set of $V$ is appropriate which is known to be suitable already in the GW case. However, for $h(x)=x^{1 /(2 k+1)}$ for different $k$ different $V$ are needed. This suggests that the nontrivial choices of $h$ relate to distinct sets of $V$.

Indeed, what occurs in the above examples extends to other functions $h$ for which a strictly monotonous inverse function $\eta$ exists,

$$
h(\eta(x))=x, \quad \eta(y)>\eta(x) \text { for } y>x .
$$


In terms of operators one then has a corresponding hermitian function $E$ with $H(E(X))=$ $X$. This allows us to introduce the general normalization-type definition of $V$,

$$
V=-D_{\mathrm{W}}^{(\eta)}\left(\sqrt{D_{\mathrm{w}}^{(\eta) \dagger} D_{\mathrm{W}}^{(\eta)}}\right)^{-1}
$$

with

$$
D_{\mathrm{W}}^{(\eta)}=i E\left(\frac{1}{2 i} \sum_{\mu} \gamma_{\mu}\left(\nabla_{\mu}-\nabla_{\mu}^{\dagger}\right)\right)+E\left(\frac{r}{2} \sum_{\mu} \nabla_{\mu}^{\dagger} \nabla_{\mu}\right)+E(m \mathbb{1}),
$$

where the function $E$ of the operators (which are hermitian) is well-defined. This definition of $V$ is to be inserted into the general expression for $D$,

$$
D=-i V^{\frac{1}{2}} a^{-1} H\left(\frac{1}{2 i}\left(V^{\frac{1}{2}}-V^{-\frac{1}{2}}\right) W\left(\frac{1}{2}\left(V+V^{\dagger}\right)\right)\right),
$$

which is (4.39) up to a factor $a^{-1}$. In the free case for this $V$ at the corners of the Brillouin zone one gets $\tilde{V}=-1$ and at zero

$$
\tilde{V} \rightarrow 1-\frac{i}{|\eta(m)|} \tilde{E}\left(a \sum_{\mu} \gamma_{\mu} p_{\mu}\right) \quad \text { for } \quad a \rightarrow 0 .
$$

Requiring $\tilde{W}(-1) \neq 0$, because of the monotony of $E(X)$ doublers are suppressed for $-2 r<m<0$ as usual. Since $H(E(X))=X$, putting $\tilde{W}(1)=2|\eta(m)|$ the correct limit of the propagator is obtained in this quite general way.

We note that the strict use of hermitian functions of hermitian operators in (4.53) with (4.54) is what makes it applicable in a general way. In contrast to this the formulation of (3.7) with (3.8) appears restricted to the choice $h(x)=x^{1 /(2 k+1)}$. Treating the latter choice with the more satisfactory form (4.53) with (4.54), instead of (4.43) one gets

$$
\tilde{V}(\kappa) \rightarrow 1-i\left(\frac{a}{|m|}\right)^{2 k+1}\left(\sum_{\mu} \gamma_{\mu} p_{\mu}\right)^{2 k+1} \quad \text { for } \quad a \rightarrow 0,
$$

with different position of the factor $i$. On the other hand, instead of the first relation in (4.44) one then has

$$
\tilde{W}(1)=2|m|^{2 k+1}, \quad \tilde{W}_{k}(1)=2^{1 /(2 k+1)}|m|,
$$

in which the factors $(-1)^{k}$ and $a^{-1}$ of (4.44) do not occur. The latter factor is now incorporated in the definition (4.55) of $D$ while the former one is accounted for by the different position of $i$ in (4.57). Thus for the choice $h(x)=x^{1 /(2 k+1)}$, for which both formulations of $V$ are applicable, one gets only minor differences in the representations.

\subsection{Chirality of $D^{-1}$}

If the inverse $D^{-1}$ exists one may multiply (11.1) from both sides with it, which gives

$$
\left\{D^{-1}, \gamma_{5}\right\}=\rho^{-1} \text {. }
$$


This relation indicates that the propagator is chiral up to a local contact term, which is desirable to get the Ward identities of chiral symmetry in the continuum limit. In case of Fujikawa's operator this relation becomes

$$
\left\{D^{-1}, \gamma_{5}\right\}=2 a_{0}^{2 k+1}\left(D \gamma_{5}\right)^{2 k}=a_{0}\left(2(1-V)^{2 k}(-V)^{-k}\right)^{1 /(2 k+1)},
$$

so that the locality of interest follows from that of $D$. For the example of the Dirac operator (4.42), which contains these operators as well as our constructions (4.1) and (4.24) as special cases, we obtain

$$
\left\{D^{-1}, \gamma_{5}\right\}=\left(2(1-V)^{2 k}(-V)^{-k}\right)^{1 /(2 k+1)} W_{k}\left(\frac{1}{2}\left(V+V^{\dagger}\right)\right)^{-1} .
$$

Since this differs from (4.60) only in that $a_{0}$ is replaced by the function $W_{k}\left(\frac{1}{2}\left(V+V^{\dagger}\right)\right)^{-1}$, the additional requirement is that the properties of $W$ should be such that the product on the r.h.s. of (4.61) remains local.

The above strategy to relate the locality of $\left\{D^{-1}, \gamma_{5}\right\}$ to that of $D$ relies on the introduction of an inverse function to $D=F(V)$,

$$
V=\mathbb{1}-I(D),
$$

with which from the basic relation (1.4) one generally obtains

$$
\left\{D^{-1}, \gamma_{5}\right\}=\gamma_{5} I(D) D^{-1}
$$

In the special case of (4.60) according to (3.5) one has $I(D)=2 a_{0}^{2 k+1}\left(D D^{\dagger}\right)^{k} D$ so that $I(D) D^{-1}=2 a_{0}^{2 k+1}\left(D D^{\dagger}\right)^{k}$. Analogously in the general case it is seen that $I(D)$ must be such that locality of $D$ implies that of $I(D) D^{-1}$.

To study this in more detail we consider the subclass of Dirac operators for which $I(D) D^{-1}=(\mathbb{1}-V) D^{-1}$ is a polynomial of $D D^{\dagger}$,

$$
I(D) D^{-1}=\sum_{\nu=0}^{N} \mathcal{C}_{\nu}\left(D D^{\dagger}\right)^{\nu}
$$

with real coefficients $\mathcal{C}_{\nu}$. In terms of the spectral functions this means that

$$
\left(1-\mathrm{e}^{i \varphi}\right) f\left(\mathrm{e}^{i \varphi}\right)^{-1}=\sum_{\nu=0}^{N} \mathcal{C}_{\nu}\left(f\left(\mathrm{e}^{i \varphi}\right) f\left(\mathrm{e}^{i \varphi}\right)^{*}\right)^{\nu},
$$

which inserting (4.12), $f\left(\mathrm{e}^{i \varphi}\right)=\mathrm{e}^{i(\varphi-\pi) / 2} g(\varphi)$, becomes

$$
2 s_{0}(\varphi)=\sum_{\nu=0}^{N} \mathcal{C}_{\nu} g(\varphi)^{2 \nu+1}, \quad s_{0}=\sin \frac{\varphi}{2}
$$

To obtain $D$ by inserting $f$ into (2.3), we have to determine $g$, which according to 4.66) requires to solve the algebraic equation

$$
\sum_{\nu=0}^{N} \mathcal{C}_{\nu} g^{2 \nu+1}-2 s_{0}=0
$$


The solution of (4.67) is trivial if only one of the coefficients $\mathcal{C}_{\nu}$ is different from zero, which gives Fujikawa's proposal.

In order to derive a more general explicit solution we consider the case where the two coefficients $\mathcal{C}_{0}$ and $\mathcal{C}_{1}$ are nonzero. We then have the cubic equation

$$
g^{3}+3 p g+2 q=0, \quad p=\frac{\mathcal{C}_{0}}{3 \mathcal{C}_{1}}, \quad q=-\frac{s_{0}}{\mathcal{C}_{1}} .
$$

Requiring $\mathcal{C}_{0}>0$ and $\mathcal{C}_{1}>0$ it follows that $p^{3}+q^{2}>0$, which implies that one gets the real solution

$$
g=\sqrt[3]{-q+\sqrt{q^{2}+p^{3}}}+\sqrt[3]{-q-\sqrt{q^{2}+p^{3}}}
$$

Thus in more detail we arrive at

$$
g=h\left(\frac{s_{0}}{\mathcal{C}_{1}}\right)=\sqrt[3]{\frac{s_{0}}{\mathcal{C}_{1}}+\sqrt{\left(\frac{s_{0}}{\mathcal{C}_{1}}\right)^{2}+\left(\frac{\mathcal{C}_{0}}{3 \mathcal{C}_{1}}\right)^{3}}}+\sqrt[3]{\frac{s_{0}}{\mathcal{C}_{1}}-\sqrt{\left(\frac{s_{0}}{\mathcal{C}_{1}}\right)^{2}+\left(\frac{\mathcal{C}_{0}}{3 \mathcal{C}_{1}}\right)^{3}}},
$$

where the new nontrivial function $h$ has the required properties of being odd and strictly monotonous. The associated inverse function according to (4.68) is $\eta(g)=\frac{1}{2}\left(g^{3}+\frac{\mathcal{C}_{0}}{\mathcal{C}_{1}} g\right)$. In the related operator expression (4.55) for $D$ now $H$ is of form (4.70) with $s_{0}$ replaced by $\frac{1}{2 i}\left(V^{\frac{1}{2}}-V^{-\frac{1}{2}}\right)$ and $W$ is constant, $W=1 / \mathcal{C}_{1}$. The condition for the usual normalization of the propagator in the continuum limit thus becomes $1 / \mathcal{C}_{1}=2|\eta(m)|$ or $\mathcal{C}_{1}|m|^{3}+\mathcal{C}_{0}|m|=1$.

It is now seen how one could proceed to still more general cases. Firstly, one could solve (4.67) for more coefficients. Secondly, one could consider more complicated expressions on the r.h.s. of (4.64).

It should, furthermore, be noted that instead of the indicated strategy in many cases it may be more convenient to analyze directly

$$
\left\{D^{-1}, \gamma_{5}\right\}=-i a\left(V^{\frac{1}{2}}-V^{-\frac{1}{2}}\right) H\left(\frac{1}{2 i}\left(V^{\frac{1}{2}}-V^{-\frac{1}{2}}\right) W\left(\frac{1}{2}\left(V+V^{\dagger}\right)\right)\right)^{-1}
$$

which follows from the general form (4.55) of $D$. Generally the desired locality obviously puts some additional conditions on the choices of the functions $H$ and $W$, however, leaving still much freedom.

\section{Weyl operators and chiral gauge theories}

The chiral projection operators implicit in the overlap formalism of Narayanan and Neuberger [8] and used in the formulation of Lüscher [3] in our notation read

$$
P_{ \pm}=P_{ \pm}^{\dagger}=\frac{1}{2}\left(1 \pm \gamma_{5}\right) \mathbb{1}, \quad \tilde{P}_{ \pm}=\tilde{P}_{ \pm}^{\dagger}=\frac{1}{2}\left(1 \pm \gamma_{5} V\right) \mathbb{1}
$$


Obviously only $\gamma_{5}$ and $V$ are involved in them and we can start with them only requiring generalized chiral symmetry. From condition (1.4) we get the identity $D=\frac{1}{2}\left(D-\gamma_{5} D \gamma_{5} V\right)$ and inserting $\gamma_{5}=P_{+}-P_{-}$and $\gamma_{5} V=\tilde{P}_{+}-\tilde{P}_{-}$into it we obtain

$$
D=P_{+} D \tilde{P}_{-}+P_{-} D \tilde{P}_{+} .
$$

With this we have the relations

$$
P_{ \pm} D \tilde{P}_{\mp}=D \tilde{P}_{\mp}=P_{ \pm} D,
$$

which generalize the expressions for the Weyl operators in terms of the Dirac operator familiar in continuum theory.

With respect to possible forms of (5.3) one should be aware of the fact that the relations

$$
P_{ \pm} \gamma_{5}= \pm \gamma_{5} \mathbb{1}, \quad \gamma_{5} V \tilde{P}_{\mp}=\mp \tilde{P}_{\mp},
$$

allow to absorb parts of $D$. In the special case of the Dirac operator (1.3), with (5.4) one gets $P_{+} \rho(1-V) \tilde{P}_{-}=2 \rho P_{+} \tilde{P}_{-}$, which relates the different forms of the chiral determinant in Ref. [3] and in Ref. [8], commented on in Refs. [16, 17]. Considering the general class of operators $D$ here, we have to observe that (5.3) is the generally valid form and that modifications by (5.4) depend on the particular choice of $D$. Furthermore, we also note that for any operator $C$ satisfying $\gamma_{5} C-C \gamma_{5} V=0$ one gets $P_{ \pm} D \tilde{P}_{\mp}=P_{ \pm}(D+C) \tilde{P}_{\mp}$, which provides another possibility to modify (5.3).

For the numbers of the degrees of freedom $\operatorname{Tr} P_{+}$and $\operatorname{Tr} \tilde{P}_{-}$of the Weyl fermions in $P_{+} D \tilde{P}_{-}$one gets from (5.1)

$$
\operatorname{Tr} P_{+}-\operatorname{Tr} \tilde{P}_{-}=\frac{1}{2} \operatorname{Tr}\left(\gamma_{5} V\right),
$$

which obviously depends only on $V$ for the whole class and agrees with the result (2.14) for the index of the Dirac operator $D$.

The degrees of freedom are exhibited in more detail representing the projections by

$$
P_{+}=\sum_{j} u_{j} u_{j}^{\dagger}, \quad \tilde{P}_{-}=\sum_{k} \tilde{u}_{k} \tilde{u}_{k}^{\dagger}, \quad u_{i}^{\dagger} u_{j}=\delta_{i j}, \quad \tilde{u}_{k}^{\dagger} \tilde{u}_{l}=\delta_{k l},
$$

with basis vectors relating the degrees of freedom to the representation in full space. Associating Grassmann variables $\bar{\chi}_{j}$ and $\chi_{k}$ to the degrees of freedom, the fermion field variables then get

$$
\bar{\psi}=\sum_{j} \bar{\chi}_{j} u_{j}^{\dagger}, \quad \psi=\sum_{k} \tilde{u}_{k} \chi_{k} .
$$

With this for $\operatorname{Tr} \tilde{P}_{-}=\operatorname{Tr} P_{+}$correlation functions are given by

$$
\begin{gathered}
\int \prod_{l}\left(\mathrm{~d} \bar{\chi}_{l} \mathrm{~d} \chi_{l}\right) \exp (-\bar{\psi} D \psi) \psi_{n(1)} \bar{\psi}_{n\left(r_{1}\right)} \psi_{n(2)} \bar{\psi}_{n\left(r_{2}\right)} \ldots \psi_{n(f)} \bar{\psi}_{n\left(r_{f}\right)}= \\
\sum_{s_{1}, \ldots, s_{f}} \epsilon_{s_{1} s_{2} \ldots s_{f}}\left(\tilde{P}_{-} D^{-1} P_{+}\right)_{n\left(s_{1}\right) n\left(r_{1}\right)} \ldots\left(\tilde{P}_{-} D^{-1} P_{+}\right)_{n\left(s_{f}\right) n\left(r_{f}\right)} \operatorname{det} M
\end{gathered}
$$


where the matrix $M$ occurring in the chiral determinant is

$$
M_{j k}=u_{j}^{\dagger} D \tilde{u}_{k}
$$

We thus have arrived at the usual basic relations of chiral gauge theories using only generalized chiral symmetry and not the GW relation.

\section{Conclusions}

We have shown that generalized chiral symmetry provides the general principle for the description of massless fermions on the lattice and for the formulation of chiral gauge

theories there, while the GW relation and its generalization by Fujikawa occur only as special cases in this context. It has turned out that this principle gives a much larger class of operators than one has in the GW and Fujikawa cases. We have given a general construction of these operators and an associated realization of the basic unitary operator $V$ involved. The index of the Dirac operator has been found to depend only on $V$.

\section{Acknowledgement}

I wish to thank Michael Müller-Preussker and his group for their kind hospitality.

\section{References}

[1] P.H. Ginsparg and K.G. Wilson, Phys. Rev. D 25 (1982) 2649.

[2] M. Lüscher, Phys. Lett. B 428 (1998) 342.

[3] M. Lüscher, Nucl. Phys. B 549 (1999) 295; Nucl. Phys. B 568 (2000) 162.

[4] K. Fujikawa, Nucl. Phys. B 589 (2000) 487.

[5] K. Fujikawa, M. Ishibashi and H. Suzuki Phys.Lett. B 538 (2002) 197.

[6] T.-W. Chiu, Phys. Rev. D 58 (1998) 074511.

[7] P. Hasenfratz, V. Laliena, F. Niedermayer, Phys. Lett. B 427 (1998) 125.

[8] R. Narayanan and H. Neuberger, Phys. Rev. Lett. 71, (1993) 3251; Nucl. Phys. B 412 (1994) 574; Nucl. Phys. B 443 (1995) 305.

[9] W. Kerler, hep-lat/0202015, to appear in JHEP.

[10] M.F. Atiyah and I.M. Singer, Ann. of Math. 87 (1968) 546, Section 5. 
[11] H. Neuberger, Phys. Lett. B 417 (1998) 141; Phys. Lett. B 427 (1998) 353.

[12] T.-W. Chiu, Phys. Lett. B 521 (2001) 429.

[13] W. Kerler, Phys. Lett. B 510 (2001) 325.

[14] T.-W. Chiu, Nucl. Phys. B 588 (2000) 400.

[15] W. Kerler, Int. J. Mod. Phys. A 16 (2001) 3117.

[16] H. Neuberger, in Lattice Fermions and Structure of the Vacuum, V. Mitrjushkin and G. Schierholz (eds.), (Kluwer Academic Publishers, 2000) p. 113.

[17] M. Golterman, Nucl. Phys. Proc. Suppl. 94 (2001) 189. 